\title{
POSTER PRESENTATION - 2
}

\section{MANAGING LABORATORY SETUP AND PRACTICES}


P2. 1

\title{
Improvement of Turnaround Time in the Clinical Laboratory
}

\author{
Leena Dash
}

Consultant Biochemist, Wockhardt Hospitals, Bangalore

Turnaround time of a lab (TAT) is very often one of the indicators by which the quality of a lab is determined. Besides this, it is very important for critical decisions in patient care. As part of our quality improvement programmes, a project was undertaken to improve the turnaround time of some commonly performed tests, which were also of utmost importance for patient care.

The tests monitored were Random blood sugar, BUN (blood urea nitrogen), creatinine, electrolytes and troponin T. The turnaround time for these tests varied from 2 to 2.5 hours, and there was a lot of variability before the project was undertaken. A structured analysis of the causes of delay was carried out. Specific interventions were put, based on these analyses. With interventions, it was possible to process $80 \%$ of the samples in 1 hour. The number of outliers also decreased after the designed interventions were adopted. Cardiac troponin T has stipulated guidelines by NACB (National Academy of Clinical Biochemistry), to be followed, with regard the turnaround time. The project focused on cardiac T separately and the TAT was brought down for almost $60 \%$ of the samples.

Analysis of the interventions, helped to determine which was most effective in improving the process. Recommendations were made as to implement the processes so as to improve the efficiency and quality of the lab. Hence this project, besides improving our lab efficiency, was useful for the hospital as it enabled quicker decision making for patient care

P2.2

\section{Impact of Preanalytical Errors Occuring in Sample Collection}

\author{
Deepti Gautam 1, Praduman Singh², B. K. Agrawal', A. K. Harith' \\ ${ }^{1}$ Gandhi Medical College, Bhopal, ${ }^{2}$ Armed Forces Medical College, Pune.
}

Evaluation and observation of types and frequencies of errors occur in sample collection and processing in large hospital. and to study the impact of collecting samples in vacutainer on these pre analytical errors. And also the impact of collecting samples in vacutainer on these pre analytical errors.

4002 consecutive samples were included in the study which was designed to monitor the types of preanalytical variables that occur in open (sample taken in bottles) and closed (sample taken in vacutainers) systems during sample collection. There were no exclusion criteria.

Among a total of 4002 specimens for 20,745 analyses, 1,297 findings were confirmed as preanalytical mistakes; this was a relative frequency of $32.4 \%(1,297 / 20,745)$. In the Open system, the relative 
Indian Journal of Clinical Biochemistry, December 2007, Vol. 22 (Supl.)

frequency of mistakes was significantly higher from the frequency of closed system $(P<0.001)$. Considering the preanalytical mistakes, the most common faults depended on inaccurate procedures for sample collection, including inappropriate quantity of specimen $(47.2 \%)$, and utilization of an inappropriate container $(0.25 \%)$. Other frequent mistakes were specimen lost / not received $(0.5 \%)$ and one or more mandatory ID missing $(2.4 \%)$, incorrect specimen collection time $(3.4 \%)$, hemolysis $(2.5 \%)$, lipemia $(0.05 \%)$ and received leaking specimen $(0.09 \%)$ causing a risk of delay; an erroneous diagnosis; and inappropriate treatment. The laboratory including laboratory reception area, of course, has no control over such defects.

Preanalytical variations should be considered as a global problem and multidisciplinary approach should be adopted. At present there are no inbuilt quality checks to access the preanalytical variation. Such check systems have to be evolved and followed universally if we want to do justice to our patients.

P2.3

\title{
Metabolic screening - Results from Metabolic Disorders Laboratory, Amrita Institute of Medical Sciences \& Research Center, Kochi, Kerala, India
}

\author{
Sumitra K, Rajesh PC, Kannan Vaidyanathan
}

Metabolic Disorders Laboratory, Dept of Biochemistry, Amrita Institute of Medical Sciences \& Research Center, Kochi, Kerala.

1575 patients were screened for different metabolic disorders during the time period from October 2006 to July 2007. Following disorders were considered - Aminoacidurias, organic aciurias, carbohydrate metabolism disorders, mucopolysaccharidoses, congenital adrenal hyperplasia, pheochromocytoma, hyperhomocysteinemia and porphyrias.

Patients admitted to Amrita Institute of Medical Sciences, Kochi and other hospitals in Kerala with signs and symptoms suggestive of metabolic disorder were testd.

$3 \mathrm{ml}$ of blood was taken in EDTA tube \& plasma was used for ELISA and HPLC of amino acids. $20 \mathrm{ml}$ fresh urine was collected for other tests. Organic acid HPLC was done on urine samples. Aminoacidurias and organic acidurias were identified by a combination of urine screening followed by high performance liquid chromatography (HPLC). Carbohydrate metabolism disorders were identified by special screenign tests followed by thin layer chromatography. Mucopolysaccharides and porphyria were identified by manual screening followed by semi-quantitative tests. Homocysteine, ADA, VMA and 17 hydroxy progesterone assays were done by ELISA.

We detected a high incidence of organic acidurias in this population. Among organic acidurias, higher prevalence of propionic aciduria and methylmalonic aciduria were seen. 2 cases of maple syrup urine disease, one case of isovaleric aciduria, one alkaptonuria and one case of hawkinsuria were also detected. Seven cases were detected with mildly elecated arginine levels. Higher prevalence of hyperhomocysteinemia (50 cases out of 184 cases screened) and congenital adrenal hyperplasia, assessed by 17 alpha hydroxy progesterone level (47 cases out of 187 cases screened) were seen. One phynyl 
Indian Journal of Clinical Biochemistry, December 2007, Vol. 22 (Supl.)

ketonuria was confirmed by amino acid quantification. Another case was detected to have hyperphenylalaninemia. Hyperglycinemia was most often associated with organic aciduria in our population.

We have detected a high incidence of metabolic disorders in our population. Specifically, high incidence of hyperhomocysteinemia, congenital adrenal hyperplasia and organic acidurias were detected. Prompt identification of disorder has helped to institute appropriate treatment measures.

P2.4

\section{Thyroid Profile Patterns in a Tertiary Care Setting}

Monika Chadha, Gurpreet Singh

SPS Apollo Hospitals, Ludhiana

Hypothyroidism (hypoT) and hyperthyroidism (hyperT) are being increasingly screened for and diagnosed in the current clinical scenario in India. Often TSH alone is being used to diagnose cases. The prevalence of subclinical hypothyroidism (s-hypoT) mistaken for true hypoT in this population is not known. It is also unclear if there is any benefit of routinely using Free T3/T4 assays in place of Total T3/T4. This study was undertaken to address these questions.

A retrospective analysis of thyroid function tests performed at our centre on 1992 consecutive patients from January 2006 till July 2007 was undertaken. Competitive immunoassay was used for T3/T4 and immunometric assay for TSH by chemiluminescence. The characteristics of thyroid function tests in the population were described and correlation between TSH and T4 assays sought.

Based on combined use of T3/T4 along with TSH, 84.1\% cases were found to be euthyroid (euT), 9.64\% hypoT and $6.18 \%$ hyperT. In contrast, based on use of TSH alone, $72 \%$ of patients were euT, $16.9 \%$ cases hypoT and $13.1 \%$ cases hyperT - suggesting over-diagnosis of both hypoT and hyperT. Of the patients with elevated TSH, Total T4 identified $60 \%$ cases as s -hypoT and $40 \%$ as true hypoT. Free T4 identified $70.2 \%$ cases as $s$-hypoT and $29.8 \%$ as true hypoT. Negative correlation of TSH with Free T4 $(r=-0.37$, $\mathrm{p}<0.01)$ was significantly better as compared to Total T4 ( $r=-0.26, p<0.01)$.

Using TSH alone to diagnose hypoT or hyperT results in significant over-diagnosis of these conditions. Therefore T3/T4 assays should be used to confirm such suspected cases. Additionally, Free T4 correlates better with TSH and is more useful in differentiating s-hypoT from true hypoT. Therefore routinely measuring Free T4 instead of Total T4 is beneficial. 